\title{
THE THEORY OF $p$-SPACES WITH AN APPLICATION TO CONVOLUTION OPERATORS $\left({ }^{1}\right)$
}

\begin{abstract}
BY
CARL HERZ( $\left.{ }^{2}\right)$

Abstract. The class of $p$-spaces is defined to consist of those Banach spaces $B$ such that linear transformations between spaces of numerical $L_{p}$-functions naturally extend with the same bound to $B$-valued $L_{p}$-functions. Some properties of $p$-spaces are derived including norm inequalities which show that 2-spaces and Hilbert spaces are the same and that $p$-spaces are uniformly convex for $1<p<\infty$. An $L_{q}$-space is a $p$-space iff $p \leqq q \leqq 2$ or $p \geqq q \geqq 2$; this leads to the theorem that, for an amenable group, a convolution operator on $L_{p}$ gives a convolution operator on $L_{q}$ with the same or smaller bound. Group representations in $p$-spaces are examined. Logical elementarity of notions related to $p$-spaces are discussed.
\end{abstract}

0. Introduction. Let $R$ designate the field of real or complex numbers. We denote by $\mathscr{B}$ the category whose objects are complete normed linear spaces over $R$ and whose morphisms are the bounded $R$-linear transformations of norm $\leqq 1$. Thus $\mathscr{B}(B, C)$ is the unit ball of $\operatorname{HOM}(B, C)$, the latter being the Banach space of all bounded $R$-linear transformations from $B$ to $C$. The endofunctor $C \mapsto \operatorname{HOM}(B, C)$ has a left adjoint $A \mapsto A \otimes B$. In more concrete terms, the tensor product may be viewed this way: each element $t \in A \otimes B$ has a representation $t=\sum_{1}^{\infty} a_{n} \otimes b_{n}$ where $\left\{a_{n}\right\} \subset A,\left\{b_{n}\right\} \subset B$ and $\|t\| \leqq \sum\left\|a_{n}\right\|\left\|b_{n}\right\|<\infty$, indeed $\|t\|$ is the infimum of $\sum\left\|a_{n}\right\|\left\|b_{n}\right\|$ taken over all representations. The concrete viewpoint is given only as a heuristic crutch.

Suppose $(\mu)$ is a measure space and $1 \leqq p<\infty$. There is an obvious endofunctor $L_{p}(\mu ; \cdot)$ of the category $\mathscr{B}$ and a natural epimorphism

$$
\varepsilon_{p}(\mu): L_{p}(\mu ; R) \otimes \cdot \rightarrow L_{p}(\mu ; \cdot) .
$$

Suppose that $(\nu)$ is also a measure space and $\varphi: L_{p}(\mu ; R) \rightarrow L_{p}(\nu ; R)$ is a morphism.

Received by the editors April 8, 1970.

AMS 1969 subject classifications. Primary 2265, 4255, 4725; Secondary 2850.

Key words and phrases. Category of Banach spaces, tensor product, $L_{p}$-space, $p$-space, locally compact group, representation, representative function, regular representation on $L_{p}$, convolution operator, measure space, Bochner integral, negative-definite function, ultraproduct.

${ }^{1}$ ) The applications to harmonic analysis on groups were announced at the Summer Institute of the University of Warwick, 1968.

$\left(^{2}\right)$ Research for this paper was sponsored by the National Science Foundation, Contract GP 11767, and Army Grant DA-ARO-D-31-124-G866.

Copyright (C) 1971, American Mathematical Society 
Given an object $B$ of $\mathscr{B}$ we may ask whether there exists a commutative diagram

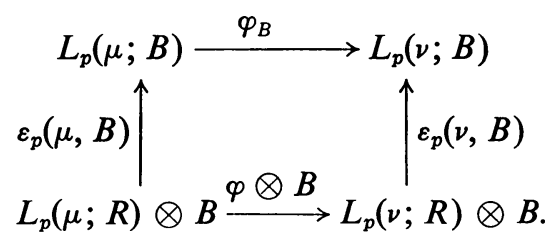

Since $\varepsilon_{p}(\mu, B)$ is an epimorphism, if the morphism $\varphi_{B}$ exists it is unique.

Write $\mathscr{L}_{p}$ to designate the full subcategory of $\mathscr{B}$ whose objects are the $L_{p}(\mu, R)$ spaces. We shall say that an object $B$ of $\mathscr{B}$ is a $p$-space if for each $\varphi \in \mathscr{L}_{p}$ there is a morphism $\varphi_{B}$ such that the above diagram is commutative. The full subcategory of $\mathscr{B}$ whose objects are $p$-spaces will be denoted by $\mathscr{B}_{p}$.

There is an equivalent characterization of $p$-spaces which is useful in the applications we have in mind. Given a Banach space $B$, write $B^{\prime}=\operatorname{HOM}(B, R)$ for the conjugate space. Then there is a canonical morphism $B \otimes B^{\prime} \rightarrow R$, called the "trace," which has the effect $b \otimes b^{\prime} \rightarrow\left\langle b, b^{\prime}\right\rangle=$ the value of $b^{\prime}$ at $b$. ( $B \otimes B^{\prime}$ may be viewed as the space of trace-class operators on $B$.) The trace induces a transformation

$$
(E \otimes B) \otimes\left(F \otimes B^{\prime}\right) \stackrel{c_{B}}{\longrightarrow} E \otimes F
$$

which is natural in $E$ and $F$, called "tensor contraction," its effect is $(e \otimes b) \otimes\left(f \otimes b^{\prime}\right) \rightarrow\left\langle b, b^{\prime}\right\rangle e \otimes f$. The examples of interest here are $E=L_{p}(\mu ; R)$, $F=L_{p^{\prime}}(\nu ; R)$ where $p^{\prime}$ is the conjugate index to $p: 1 / p+1 / p^{\prime}=1$.

THEOREM 0. A Banach space $B$ is a p-space iff for each pair $\mu, \nu$ of measure spaces there is a commutative diagram

$$
\begin{aligned}
L_{p}(\mu ; B) & \otimes L_{p^{\prime}}\left(\nu ; B^{\prime}\right) \stackrel{\gamma_{B}}{\longrightarrow} L_{p}(\mu ; R) \otimes L_{p^{\prime}}(\nu ; R) \\
\varepsilon_{p}(\mu, B) & \otimes \uparrow \varepsilon_{p^{\prime}}\left(\nu, B^{\prime}\right) \\
{\left[L_{p}(\mu ; R) \otimes B\right] } & \otimes\left[L_{p^{\prime}}(\nu ; R) \otimes B^{\prime}\right]
\end{aligned}
$$

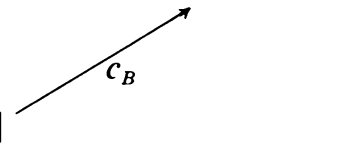

where $c_{B}$ is tensor contraction.

Heuristically, the way to picture Theorem 0 is this. Suppose $\mu$ is a Radon measure on a locally compact space $X$ and $v$ is a Radon measure on a locally compact space $Y$. Let $u: X \rightarrow B$ and $v: Y \rightarrow B^{\prime}$ be continuous functions of compact support. Define $\varphi: X \times Y \rightarrow R$ by $\varphi(x, y)=\langle u(x), v(y)\rangle$ where $\langle$,$\rangle is the pairing of B$ and $B^{\prime}$. The condition that $B$ is a $p$-space is that $\varphi$ represent an element of $L_{p}(\mu ; R) \otimes L_{p^{\prime}}(\nu ; R)$ where norm satisfies $\|\varphi\| \leqq\|u\|_{p}\|v\|_{p^{\prime}}$

The fundamental theorem on the Bochner integral [1] is that for all measure spaces $\mu$ the transformation

$$
\varepsilon(\mu): L_{1}(\mu ; R) \otimes \cdot \rightarrow L_{1}(\mu ; \cdot)
$$

is a natural isomorphism. Thus $\mathscr{B}=\mathscr{B}_{1}$, and we may restrict our attention to $\mathscr{B}_{p}$ 
for $1<p<\infty$. The only other complete characterization available is $\mathscr{B}_{2}=\mathscr{L}_{2}$ $=$ Hilbert spaces. The main analytical result in this paper is

THEOREM 1. Suppose $1<p<\infty$ and $1 \leqq q \leqq \infty$. If $p \leqq q \leqq 2$ or $p \geqq q \geqq 2$ then $\mathscr{L}_{q} \subset \mathscr{B}_{p}$. In all other cases the only $\mathscr{L}_{q}$-spaces which are p-spaces are 0 and $R$.

The point of insisting on a categorical approach is that the genuine analytic content of Theorem 1 involves only finite-dimensional Banach spaces and rests on a result of Paul Lévy, see $\$ 2$ below. Indeed, the affirmative part of Theorem 1 for real scalars could be deduced from the special case already given by Marcinkiewicz and Zygmund [7].

We have in mind applications to group representations. Let $G$ designate a locally compact group. A representation $\xi$ of $G$ consists of a complex Banach space $E(\xi)$ and a continuous homomorphism $U_{\xi}: G \rightarrow \mathrm{AUT}_{s} E(\xi)$, the group of automorphisms (isometries) of $E(\xi)$ endowed with the strong operator topology. A morphism $\xi \stackrel{h}{\rightarrow} \eta$ of representations in a morphism $E(\xi) \stackrel{h}{\rightarrow} E(\eta)$ of Banach spaces such that $U_{\eta}(x) \circ h=h \circ U_{\xi}(x)$ for each $x \in G$. We obtain a category $\operatorname{Rep}(G)$ in which sums, tensor products, etc. are easily defined in the obvious way. Isomorphism classes of representations form too fine a distinction, e.g. there is one isomorphism class of trivial representation for each isomorphism class of Banach spaces. To avoid this difficulty the following procedure is used. Given a representation $\xi$ let $E=E(\xi)$, and let $E^{\prime}=\operatorname{HOM}(E, C)$ be the conjugate Banach space. Then there is a morphism $E \otimes E^{\prime} \stackrel{\Pi(\xi)}{\longrightarrow} C_{u}(G)$, the space of bounded uniformly continuous functions on $G$ in the supremum norm, defined by $\Pi(\xi)(e \otimes f)(x)$ $=f\left(U_{\xi}(x) e\right)$. The coimage of $\Pi(\xi)$ is called the space of $\xi$-representative functions, and it is denoted by $A(\xi)$. Thus $A(\xi)$ is a Banach space whose elements are canonically identified with certain bounded uniformly continuous functions on $G$; the norm in $A(\xi)$ is the quotient norm from $E \otimes E^{\prime}$. Example. $\xi$ is trivial iff $A(\xi)$ $=$ constant functions.

For the sum of representations it is clear that addition of functions gives an epimorphism $A(\xi)+A(\eta) \rightarrow A(\xi+\eta)$.

Similarly, for the tensor product of representations, multiplication of functions gives a morphism $A(\xi) \otimes A(\eta) \rightarrow A(\xi \otimes \eta)$.

Of particular interest are the regular representations. Let $L_{p}(G ; \cdot)$ designate the functor arising from the left-invariant Haar measure on $G$. A functor $\lambda_{p}: \operatorname{Rep}(G) \rightarrow \operatorname{Rep}(G)$ is defined by putting $\lambda_{p}(\xi)$ the representation whose representation space is $E\left(\lambda_{p}(\xi)\right)=L_{p}(G ; E(\xi))$ and whose operators are given $x \rightarrow U(x)$ where $U(x) f(y)=U_{\xi}(x) f\left(x^{-1} y\right)$, the element $f \in L_{p}(G ; E(\xi))$ being viewed as a function on $G$ with values in $E(\xi)$. The representation $\lambda_{p}(C)(C$ being the trivial representation on $C$ ) is called the left-regular representation on $L_{p}$. We write $A_{p}=A\left(\lambda_{p}(C)\right.$ ). (Had we used the right-regular representation we would have gotten the same $A_{p}$.) There are two important remarks to make about the representation $\lambda_{p}(\xi)$. 
REMARK I. Given a representation $\xi$, let $\xi^{0}$ designate the trivial representation with the same representation space. Then there is a natural isomorphism $\lambda_{p}(\xi) \stackrel{T(\xi)}{\longrightarrow} \lambda_{p}\left(\xi^{0}\right)$ of representations given by $T(\xi) f(y)=U_{\xi}\left(y^{-1}\right) f(y)$ for $f \in L_{p}(G ; E(\xi))$.

RemarK II. Multiplication of functions gives a morphism $A_{p} \otimes A(\xi) \rightarrow A\left(\lambda_{p}(\xi)\right)$. The relevance of $p$-spaces in representation theory is a consequence of

LeMma 0 . If $B \neq 0$ is a $p$-space then $A\left(\lambda_{p}(B)\right)=A_{p}$.

Proof. Recall that $A(\xi)$ is in general defined as the coimage of $E(\xi) \otimes E^{\prime}(\xi) \stackrel{\Pi(\xi)}{\longrightarrow} C_{u}(G)$. Let $L_{p}(G ; B) \otimes L_{p^{\prime}}(G ; B) \stackrel{\gamma_{B}}{\longrightarrow} L_{p}(G: C) \otimes L_{p^{\prime}}(G ; C)$ be the morphism of Theorem 0 . Then

$$
\Pi\left(\lambda_{p}(B)\right)=\Pi\left(\lambda_{p}(C)\right) \circ \gamma_{B}
$$

Since $\gamma_{B}$ is an extremal epimorphism, taking coimages gives the desired equality.

Combining this last result with Remarks I and II gives

THEOREM A. If $\xi$ is a representation in a p-space $E(\xi)$, then multiplication of functions gives a morphism $A_{p} \otimes A(\xi) \rightarrow A_{p}$.

Taking $\xi=\lambda_{p}(\boldsymbol{C})$ we get

COROLlary. $A_{p}$ is a Banach algebra under pointwise addition and multiplication of functions.

A deeper result which depends on Theorem 1 is

THEOREM B. If $p \leqq q \leqq 2$ or $p \geqq q \geqq 2$ then multiplication of functions gives $a$ morphism $A_{p} \otimes A_{q} \rightarrow A_{p}$.

Proof. Take $\xi=\lambda_{q}(\boldsymbol{C})$ in Theorem A.

For amenable groups Theorem B has some powerful implications. One can show that the conjugate Banach space to $A_{p}$ is canonically isomorphic to $\mathrm{CONV}_{p}$, the operators on $L_{p}(G ; C)$ which commute with right-translations. The result is

Theorem C. Let $G$ be an amenable group and suppose $p \leqq q \leqq 2$ or $p \geqq q \geqq 2$. Then identification of functions gives a morphism $A_{q} \rightarrow A_{p}$. Dually there is a morphism $\mathrm{CONV}_{q} \rightarrow \mathrm{CONV}_{p}$, i.e. convolution operators on $L_{p}(G ; C)$ are convolution operators on $L_{q}(G ; C)$ with contraction of norms.

The details of Theorem $\mathrm{C}$ will be given elsewhere. One remark is in order. The right-regular representations give the same $A_{p}$, and one finds that if $f(x)=f\left(x^{-1}\right)$ then $f \mapsto f$ is an isomorphism of $A_{p}$ with $A_{p^{\prime}}$. For commutative groups $A_{p}=A_{p^{\prime}}$, and Theorem $\mathrm{C}$ is an easy deduction from the Riesz Convexity Theorem. On the other hand it is not known whether for any noncommutative group one has $A_{p}=A_{p^{\prime}}$ when $p \neq 2$. Thus the only known proof of Theorem $\mathrm{C}$ depends on Theorem 1 above. 
1. Preliminaries. We define a measure space $(\mu)$ to be a Boolean $\sigma$-algebra $\mathscr{M}_{\mu}$ together with a countably additive function $\mu: \mathscr{M}_{\mu} \rightarrow[0,+\infty]$ such that for $E \in \mathscr{M}_{\mu}, \mu(E)=\sup \left\{\mu(F): E \supset F \in \mathscr{M}_{\mu}, \mu(F)<\infty\right\}$ and $\mu(E)=0$ iff $E=\varnothing$, the minimum element of $\mathscr{M}_{\mu}$. For $1 \leqq p<\infty$ the functors $L_{p}(\mu ; \cdot)$ on $\mathscr{B}$ are constructed by the following procedure. Put $\mathscr{D}_{\mu}$ for the directed set whose objects $\Delta$ are finite collections of disjoint elements $D \in \mathscr{M}_{\mu}$ with $0<\mu(D)<\infty$ and whose morphisms are $\Gamma \prec \Delta$ when each element of $\Gamma$ is a union of elements of $\Delta$. Given a Banach space $B$ put $L_{p}(\mu \Delta ; B)$ for the vector space of functions $f: \Delta \rightarrow B$ with the norm $\|f\|$ $=\left\{\sum_{D \in \Delta}|f(D)|_{B}^{p} \mu(D)\right\}^{1 / p}$. If $\Gamma \prec \Delta$ there is a natural extremal monomorphism $L_{p}(\mu \Gamma ; B) \stackrel{i(\Gamma, \Delta)}{\longrightarrow} L_{p}(\mu \Delta ; B)$ defined by $f \mapsto g$ where $g(D)=f(C)$ if $\Delta \ni D \subset C \in \Gamma$ and $g(D)=0$ if $D \in \Delta$ meets no element of $\Gamma$. The inductive limit of the direct system of functors $L_{p}(\mu \Delta ; \cdot)$ is, by definition, $L_{p}(\mu ; \cdot)$. The natural "inclusions" $i(\Gamma, \Delta)$ have natural retractions $r(\Delta, \Gamma)$ where $L_{p}(\mu \Delta ; B) \stackrel{r(\Delta, \Gamma)}{\longrightarrow} L_{p}(\mu \Gamma ; B)$ is defined by $g \mapsto f$ where $f(C)=\mu(C)^{-1} \sum_{D \subset C} g(D) \mu(D)$. In the limit one has

$$
L_{p}(\mu \Delta ; \cdot) \stackrel{i(\Delta)}{\longrightarrow} L_{p}(\mu ; \cdot) \stackrel{r(\Delta)}{\longrightarrow} L_{p}(\mu \Delta ; \cdot)
$$

where $i$ is "inclusion," $r$ is "conditional expectation," and $r \circ i=\mathrm{id}$.

The projective limit of $L_{p}(\mu \Delta ; \cdot) \stackrel{r(\Delta \Gamma)}{\longrightarrow} L_{p}(\mu \Gamma ; \cdot)$ taken with $\mathscr{D}_{\mu}$ as an inverse system yields functors $\bar{L}_{p}(\mu ; \cdot)$. There are natural extremal monomorphisms $L_{p}(\mu ; \cdot) \subset \bar{L}_{p}(\mu ; \cdot)$. Moreover, if $1<p<\infty$ and $1 / p+1 / p^{\prime}=1$ there is a natural identification of $\bar{L}_{p^{\prime}}\left(\mu ; B^{\prime}\right)$ with the conjugate space of $L_{p}(\mu ; B)$; this is a triviality since conjugation takes inductive limits into projective limits. What is not banal are conditions under which $L_{p}(\mu ; B)$ and $\bar{L}_{p}(\mu ; B)$ coincide. If, however, one knows a priori that $L_{p}(\mu ; B)$ is reflexive it is immediate that it coincides with $\bar{L}_{p}(\mu ; B)$ and has $L_{p} \cdot\left(\mu ; B^{\prime}\right)$ for conjugate space; fortunately this simple remark is all that is needed here.

The definition of $L_{p}(\mu ; \cdot)$ given above is technically very convenient and sidesteps pathologies. It requires only a little care to convert other definitions into the form used here. For example, suppose $X$ is a locally compact Hausdorff space. Let $\mathscr{K}$ be the collection of compact subsets of $X$. A Radon measure on $X$ is a function $\mu: \mathscr{K} \rightarrow[0, \infty]$ with the properties: (1) $\mu(\varnothing)=0$; (2) $\mu(K) \leqq \mu(L)$ if $K \subset L$; (3) $\mu(K \cup L)=\mu(K)+\mu(L)$ if $K \cap L=\varnothing$; (4) if $K \subset M$ then for each $\varepsilon>0$ there exists $L \subset M \backslash K$ such that $\mu(M)<\mu(K)+\mu(L)+\varepsilon$. One can then prove that there exists a unique countably-additive set-function, also denoted by $\mu$, defined on the Borel field of $X$ such that $\mu(E)=\sup \{\mu(K): E \supset K \in \mathscr{K}\}$. A measure space $(\mu)$ is obtained by taking $\mathscr{M}_{\mu}$ to be the Borel sets modulo Borel sets of measure 0 . Let $\tilde{L}_{p}(\mu ; B)$ be defined as the Banach space obtained from the vector space of continuous maps $f: X \rightarrow B$ of compact support endowed with the pseudonorm $\|f\|_{p}=\left\{\int|f|_{B} d \mu\right\}^{1 / p}$. It is very easy to see that one has natural transformations

$$
L_{p}(\mu \Delta ; B) \stackrel{\tilde{i}(\Delta)}{\longrightarrow} \tilde{L}_{p}(\mu ; \cdot) \stackrel{\tilde{r}(\Delta)}{\longrightarrow} L_{p}(\mu \Delta ; \cdot)
$$


where $\tilde{r}(\Delta)$ is "conditional expectation" and $\tilde{i}(\Delta)$ arises by lifting the elements of $\Delta$ to Borel sets in $X$ and approximating the indicator function of a Borel set of finite measure in the $L_{p}$-norm by continuous functions of compact support. In the limit one has

$$
L_{p}(\mu ; \cdot) \stackrel{\tilde{\imath}}{\longrightarrow} \tilde{L}_{p}(\mu ; \cdot) \stackrel{\tilde{r}}{\longrightarrow} \bar{L}_{p}(\mu ; \cdot)
$$

where $\tilde{r} \circ \tilde{l}$ in the inclusion of $L_{p}$ in $\bar{L}_{p}$. Since $\tilde{r}$ is obviously a monomorphism, $\tilde{\imath}$ must be an isomorphism.

The particular convenience here of the given definition of $L_{p}$-spaces rests on two observations. The natural diagram (in $B$ )

$$
\begin{array}{cc}
L_{p}(\mu \Delta ; B) \stackrel{i(\Delta, B)}{\longrightarrow} & L_{p}(\mu ; B) \\
\varepsilon_{p}(\mu \Delta, B) \uparrow & \uparrow \varepsilon_{p}(\mu, B) \\
L_{p}(\mu \Delta ; R) \otimes B \stackrel{i(\Delta, R) \otimes B}{\longrightarrow} L_{p}(\mu ; R) \otimes B
\end{array}
$$

is commutative and the right-hand side is the inductive limit of the left-hand side. The natural diagram

$$
\begin{array}{cc}
L_{p}(\nu ; B) & r(\Gamma, B) \\
\varepsilon_{p}(\nu, B) \uparrow & L_{p}(\nu \Gamma ; B) \\
L_{p}(\nu ; R) \otimes B \stackrel{r(\Gamma, R) \otimes B}{\longrightarrow} & \uparrow \varepsilon_{p}(\nu \Gamma, B) \\
L_{p}(\nu \Gamma ; R) \otimes B
\end{array}
$$

is commutative, and although the left-hand side is not always the projective limit of the right-hand side (it is if $B$ is a $p$-space) the image of

$$
L_{p}(\nu ; R) \otimes B \rightarrow \operatorname{proj} \lim \left[L_{p}(\nu \Gamma ; R) \otimes B\right] \rightarrow \operatorname{proj} \lim L_{p}(\nu \Gamma ; B)=\bar{L}_{p}(\nu ; B)
$$

lies in the subspace $L_{p}(\nu ; B)$ of $\bar{L}_{p}(\nu ; B)$. It is easy to verify that any morphism $\varphi: L_{p}(\mu ; R) \rightarrow L_{p}(\nu ; R)$ is sufficiently well approximated by the morphism $r(\Gamma, R) \circ \varphi \circ i(\Delta, R)$ where $\Delta \in \mathscr{D}_{\mu}$ and $\Gamma \in \mathscr{D}_{\nu}$ that if for a given Banach space $B$ and each $\Gamma, \Delta$ there is a morphism $\varphi_{B}(\Gamma, \Delta): L_{p}(\mu \Delta ; B) \rightarrow L_{p}(\nu \Gamma ; B)$ such that

$$
\varphi_{B}(\Gamma, \Delta) \circ \varepsilon_{p}(\mu \Delta ; B)=\varepsilon_{p}(\nu \Gamma ; B) \circ\{[r(\Gamma, R) \circ \varphi \circ i(\Delta, R)] \otimes B\}
$$

then $\varphi_{B}=$ proj $\lim _{\mathscr{D}_{v}}$ ind $\lim _{\mathscr{D}_{\mu}} \varphi_{B}(\Gamma, \Delta)$ has the property that $\varphi_{B} \circ \varepsilon_{p}(\mu ; B)$ $=\varepsilon_{p}(\nu ; B) \circ(\varphi \otimes B)$. The functors $L_{p}(\mu \Delta ; \cdot)$ are naturally isomorphic to $L_{p}(\boldsymbol{m} ; \cdot)$ where $m$ is the cardinality $\Delta$. Thus the test for whether a Banach space $B$ is a $p$ space may be reduced to the consideration of morphisms $\varphi: L_{p}(\boldsymbol{m} ; R) \rightarrow L_{p}(n ; R)$ where $m$ and $n$ range over the natural numbers. A restatement of this fact is

Proposition 0. Given a pair $m, n$ of natural numbers, say for a Banach space $B$ that $B \in \mathscr{B}_{p}(m, n)$ if

$$
\sum_{i=1}^{n}\left|\sum_{j=1}^{m} M_{i j} b_{j}\right|_{B}^{p} \leqq \sum_{k=1}^{m}\left|b_{k}\right|^{p}
$$


for each $m$-tuple $b_{1}, \ldots, b_{m} \in B$ and each matrix $M$ with $m$ columns and $n$ rows having entries in $R$ such that

$$
\sum_{i=1}^{n}\left|\sum_{j=1}^{m} M_{i j} r_{j}\right|^{p} \leqq \sum_{k=1}^{m}\left|r_{k}\right|^{p}
$$

for all $m$-tuples $r_{1}, \ldots, r_{m} \in R$. Then the class of p-spaces is characterized by $\mathscr{B}_{p}$ $=\bigcap_{m, n=1}^{\infty} \mathscr{B}_{p}(m, n)$.

REMARK. As will be seen in Lemma 1 , the elements of $\mathscr{B}_{p}(2,2)$ already have the Clarkson inequalities, in particular $\mathscr{B}_{2}=\mathscr{B}_{2}(2,2)=$ Hilbert spaces. It seems unlikely, however, that $\mathscr{B}_{p}=\mathscr{B}_{p}(m, n)$ for any finite $m, n$ if $p \neq 1,2$.

Many properties of $p$-spaces can be derived from abstract arguments. In a category with pullbacks and pushouts we say that a monomorphism $i$ is an extremal monomorphism if $i=f \circ g$ and $g$ is an epimorphism imply that $g$ is an isomorphism. If $A \stackrel{i}{\rightarrow} B$ is an extremal monomorphism we say that $A$ is a subobject of $B$. In the category of Banach spaces, extremal monomorphism = isometry; hence a subspace has the same norm as the ambient space. Dually for extremal epimorphisms and quotients. Let $\mathscr{D}$ be a directed set and $\mathscr{B}$ a complete category; given a functor $F: \mathscr{D} \rightarrow \mathscr{B}$ such that whenever $x, y \in \mathscr{D}$ and $x<y$ the morphism $F(x) \rightarrow F(y)$ is an

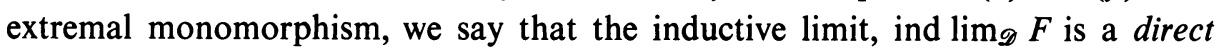
union.

If $\mathscr{B}$ is a category with a terminal object and $\mathscr{S}$ is a category with objects $a, b, z$ and morphisms $a \rightarrow b, a \rightarrow z$, then the inductive limit of a functor $F: \mathscr{S} \rightarrow \mathscr{B}$ such that $F(z)=0$ is called a "cokernel." A cokernel is a quotient, and the converse is true in some categories, e.g. Banach spaces. Inductive limits commute with each other; so that to show that an inductive limit has certain properties with respect to $L_{p}(\mu ; \cdot)$ functors it is often sufficient to consider only $L_{p}(\boldsymbol{m} ; \cdot)$ with $m$ a natural number, e.g. $L_{p}(\mu ; \cdot)$ commutes with direct unions and preserves extremal epimorphisms (view these as cokernels). Also $L_{p}(\mu ; \cdot)$ preserves extremal monomorphisms (although tensor products do not in general). The following list gives obvious results.

Proposition 1. A subspace of a p-space is a p-space.

Proposition 2. A direct union of p-spaces is a p-space.

Proposition 3. A quotient of a p-space is a p-space.

Proposition 4. $B \in \mathscr{B}_{p}$ iff $B^{\prime} \in \mathscr{B}_{p^{\prime}}$.

Proposition 5. If $B$ is a p-space so is $L_{p}(\mu ; B)$ for any measure space $(\mu)$. If $A$ and $B$ are p-spaces so is $A \oplus_{p} B$, the completion of $A+B$ for the norm $\|(a, b)\|$ $=\left\{|a|_{A}^{p}+|b|_{B}^{p}\right\}^{1 / p}$.

Some remarks. 
Remark 1. A Banach space $B$ is a $p$-space iff each finite-dimensional subspace is a $p$-space.

REMARK 2. The serious problems about $p$-spaces may be stated in terms of the range $1<p \leqq 2$.

REMARK 3. For any pair $(\mu),(\nu)$ of measure spaces there is a measure space $(\mu \times \nu)$ such that $L_{p}\left(\mu ; L_{p}(\nu, R)\right), L_{p}\left(\nu ; L_{p}(\mu ; R)\right)$, and $L_{p}(\mu \times \nu ; R)$ are canonically isomorphic. Thus spaces of the form $L_{p}(\mu ; R)$ together with their subspaces and quotients spaces are the only obvious $p$-spaces when $1<p<\infty$; no examples of $p$-spaces are known to me which are not obtained this way.

The next is an example of how abstract methods may be used.

Proof of Theorem 0. Since tensor products commute with inductive limits, to prove the existence of the required morphism

$$
\gamma_{B}: L_{p}(\mu ; B) \otimes L_{p^{\prime}}\left(\nu ; B^{\prime}\right) \rightarrow L_{p}(\mu ; R) \otimes L_{p^{\prime}}(\nu ; R)
$$

for arbitrary measure spaces $\mu, \nu$ it suffices to consider the cases $\mu=\boldsymbol{m}, \nu=\boldsymbol{n}$ where $m$ and $n$ range over the natural numbers. Now suppose $B$ is finite dimensional. If $X$ and $Y$ are finite-dimensional Banach spaces then $X \otimes Y^{\prime}$ and $\operatorname{HOM}(X, Y)$ are conjugate to each other. Thus the existence of

$$
\gamma_{B}: L_{p}(\boldsymbol{m} ; B) \otimes L_{p^{\prime}}\left(\boldsymbol{n} ; B^{\prime}\right) \rightarrow L_{p}(\boldsymbol{m} ; R) \otimes L_{p^{\prime}}(\boldsymbol{n} ; R)
$$

is equivalent to the existence of a conjugate morphism

$$
\delta_{B}: \operatorname{HOM}\left(L_{p}(\boldsymbol{m} ; R), L_{p}(\boldsymbol{n} ; R)\right) \rightarrow \operatorname{HOM}\left(L_{p}(\boldsymbol{m} ; B), L_{p}(\boldsymbol{n} ; B)\right)
$$

where $\varphi_{B}=\delta_{B}(\varphi)$ is exactly the morphism required in the definition of $p$-space. In view of Proposition 0 , we have proved Theorem 0 for finite-dimensional Banach spaces $B$. Now let $B$ be an arbitrary Banach space and $F \stackrel{i}{\rightarrow} B$ a finite-dimensional subspace; $m$ and $n$ are kept fixed in all that follows. Suppose the required morphism $\gamma_{B}$ exists. Then we get a morphism

$$
\gamma_{F, B}: L_{p}(\boldsymbol{m} ; F) \otimes L_{p^{\prime}}\left(\boldsymbol{n} ; B^{\prime}\right) \rightarrow L_{p}(\boldsymbol{m} ; R) \otimes L_{p^{\prime}}(\boldsymbol{n} ; R)
$$

given by $\gamma_{F, B}=\gamma_{B} \circ\left(L_{p}(\boldsymbol{m} ; i) \otimes L_{p^{\prime}}\left(\boldsymbol{n} ; B^{\prime}\right)\right)$. Now $B^{\prime} \stackrel{i}{\rightarrow} F^{\prime}$ is an extremal epimorphism; hence so is $L_{p^{\prime}}\left(\boldsymbol{n} ; i^{\prime}\right)$; and therefore $L_{p}(\boldsymbol{m} ; F) \otimes L_{p^{\prime}}\left(\boldsymbol{n} ; i^{\prime}\right)$ is an extremal epimorphism whose kernel is obviously contained in the kernel of $\gamma_{F, B}$. It follows that $\gamma_{F, B}$ must factor through $L_{p}(\boldsymbol{m} ; F) \otimes L_{p^{\prime}}\left(\boldsymbol{n} ; F^{\prime}\right)$; this gives the existence of $\gamma_{F}$. Conversely, if $\gamma_{F}$ exists we may define $\gamma_{F, B}$ by $\gamma_{F, B}=\gamma_{F} \circ\left(L_{p}(\boldsymbol{m} ; E) \otimes L_{p^{\prime}}\left(\boldsymbol{n} ; \boldsymbol{i}^{\prime}\right)\right)$. Assuming that $\gamma_{F}$ exists for every finite-dimensional subspace $F$, the morphism $\gamma_{B}=$ ind $\lim _{F} \gamma_{F, B}$ has the required properties.

2. Subspaces of $\mathscr{L}_{p}$-spaces. To say that a Banach space $B$ is a subspace of an $\mathscr{L}_{p}$-space is to say that there exists a measure space $(\mu)$ and an extremal monomorphism $B \stackrel{i}{\rightarrow} L_{p}(\mu ; R)$. A continuous function $\psi$ defined on a group $X$ is negative- 
definite if $\sum \psi\left(x_{i}-x_{j}\right) c_{i} \bar{c}_{j} \leqq 0$ for all finite collections, $x_{1}, \ldots, x_{n} \in X$ and $c_{1}, \ldots, c_{n} \in C$ with $\sum c_{i}=0$.

THEOREM 2. A Banach space $B$ is a subspace of an $\mathscr{L}_{p}$-space, $1 \leqq p \leqq 2$, iff $x \mapsto\|x\|^{p}$ is a negative-definite function on $B$. (Equivalently $x \mapsto \exp \left(-\|x\|^{p}\right)$ is a positive-definite function on $B$.)

COROLlaRY 1. If $p \leqq q \leqq 2$ then an $\mathscr{L}_{q}$-space is a subspace of an $\mathscr{L}_{p}$-space.

Proof of Corollary 1. A theorem of Bochner [2] states that if $\psi$ is a positivevalued negative-definite function and $0<\alpha \leqq 1$ then $\psi^{\alpha}$ is negative-definite. If $B$ is an $\mathscr{L}_{q}$-space then $\psi(x)=\|x\|^{q}$ is negative-definite by Theorem 2 ; hence $x \mapsto\|x\|^{p}$, which is $\psi^{\alpha}$ for $\alpha=p / q$, is negative-definite.

COROLlaRY 2. If $p \leqq q \leqq 2$ or $p \geqq q \geqq 2$ then $\mathscr{L}_{q} \subset \mathscr{B}_{p}$.

Proof of Corollary 2. By Propositions 1 and 2 it follows from Corollary 1 for finite-dimensional $\mathscr{L}_{q}$ spaces with $p \leqq q \leqq 2$ that $\mathscr{L}_{q} \subset \mathscr{B}_{p}$ since $\mathscr{L}_{p} \subset \mathscr{B}_{p}$ is already known (and obvious). If $p \geqq q \geqq 2$ then $\mathscr{L}_{q^{\prime}} \subset \mathscr{B}_{p^{\prime}}$ which implies $\mathscr{L}_{q} \subset \mathscr{B}_{p}$.

The necessity of the condition of Theorem 2 is banal. One has only to prove that $x \mapsto\|x\|^{p}$ is negative-definite on $L_{p}(\mu ; R)$ since the condition is obviously hereditary. On the other hand it is clearly preserved by direct unions; so it is sufficient to prove it for $L_{p}(\boldsymbol{m} ; R)$. For $x \in L_{p}(\boldsymbol{m} ; R)$ one has $\|x\|^{p}=\left|x_{1}\right|^{p}+\cdots+\left|x_{m}\right|^{p}$; and the sum of negative-definite functions is negative-definite. Therefore the only question is whether $x \mapsto|x|^{p}$ is negative-definite on $R$. Now $\psi(x)=|x|^{2}$ is obviously negativedefinite on $R$ (whether $R=\boldsymbol{R}$ or $\boldsymbol{C}$ ), and $|x|^{p}=\psi^{\alpha}(x)$ for $\alpha=p / 2 \leqq 1$.

The real version of Theorem 2 is known. For finite-dimensional real Banach spaces it was observed by the author [5] to be a consequence of a theorem of Paul Lévy $[6, \S 63]$ on symmetric stable laws in several variables. The extension to the infinite-dimensional case is due to Bretagnolle, Dacunha-Castelle, and Krivine [3]. We do not need this extension, but we wish to comment later on the proof, see $\$ 4$.

A complex Banach space $B$ is a real Banach space equipped with an automorphism $i$ such that $i^{2}=-\mathrm{id}$ and $\|\cos \theta b+\sin \theta(i b)\|=\|b\|$ for all $b \in B$ and all $\theta \in \boldsymbol{R}$. The complex case of Theorem 2 follows from the next (the condition that $x \mapsto\|x\|^{p}$ be negative-definite does not depend on whether real or complex scalars are used).

Proposition 6. Let $B$ be a complex Banach space and $B_{R}$ the same space viewed as a real Banach space. For each real morphism $B_{\boldsymbol{R}} \stackrel{\varphi}{\rightarrow} L_{p}(\mu ; \boldsymbol{R})$ there is a complex morphism $B \stackrel{\psi}{\rightarrow} L_{p}(\mu ; C)$ given by $\psi(b)=c_{p} \varphi(b)-i c_{p} \varphi(i b)$ where $c_{p}$ is a universal constant depending only on $p$. If $\varphi$ is an isometry so is $\psi$.

Proof. There is a constant $c_{p}$ such that

$$
(2 \pi)^{-1} \int_{0}^{2 \pi}\left|\operatorname{Re}\left(e^{i \theta} z\right)\right|^{p} d \theta=c_{p}^{p}|z|^{p}
$$


for each $z \in C$. Observe that for each $b \in B, \varphi\left(e^{i \theta} b\right)=\operatorname{Re}\left\{e^{i \theta} \psi(b)\right\} c_{p}^{-1}$. Since $\left\|\varphi\left(e^{i \theta} b\right)\right\| \leqq\|b\|$ for each $\theta$ it follows that

$$
\int\left|\operatorname{Re}\left\{e^{i \theta} \psi(b)\right\}\right|^{p} d \mu \leqq c_{p}^{p}\|b\|^{p} .
$$

Integrating with respect to $d \theta$ and interchanging the order of integration we get $\int|\psi(b)|^{p} d \mu \leqq\|b\|^{p}$ which is what was to be proved, since $b \rightarrow \psi(b)$ is obviously complex linear.

3. Properties of $p$-spaces. The affirmative part of Theorem 1 is a corollary of Theorem 2; the negative part follows from Lemmas 1 and 2 below. Indeed, Lemma 1 asserts that the elements of $\mathscr{B}_{p}(2,2)$ must satisfy certain inequalities on the norm that were obtained for $\mathscr{L}_{p}$-spaces by Clarkson [4]. In particular, for $1<p<\infty$, a space in $\mathscr{B}_{p}(2,2)$ is uniformly convex. Combining the Clarkson inequalities with Lemma 2 , one gets that the only $\mathscr{L}_{q}$-spaces in $\mathscr{B}_{p}(3,2)$ with $q<p \leqq 2$ or $q>p \geqq 2$ are 0 and $R$. After Lemma 3 one has the following, even for $\mathscr{B}_{p}(3,3)$.

Proposition 7. Suppose $1<p<\infty$. Then a p-space is uniformly convex, hence reflexive, and its norm function is strongly differentiable everywhere except at the origin.

The lemmas below are all based on elementary calculations using well-chosen morphisms $L_{p}(\boldsymbol{m} ; R) \rightarrow L_{p}(\boldsymbol{n} ; R)$.

Lemma 1. If $B \in \mathscr{B}_{p}$ then for all $x, y \in B$

$$
\|x+y\|^{p}+\|x-y\|^{p} \leqq 2^{r}\left(\|x\|^{p}+\|y\|^{p}\right), \quad r=\max (1, p-1) .
$$

Proof. $(\xi, \eta) \mapsto 2^{-r / p}(\xi+\eta, \xi-\eta)$ is an endomorphism of $L_{p}(\mathbf{2}, R)$.

COROLlaRY. $\mathscr{L}_{q} \cap \mathscr{B}_{p}=\{0, R\}$ unless $q$ is between $p$ and $p^{\prime}$.

Proof. Each $\mathscr{L}_{q}$-space other than 0 or $R$ contains a subspace isomorphic to $L_{q}(2, R)$. Hence it suffices to show that $L_{q}(2, R) \notin \mathscr{B}_{p}$. Take $x=(1,1)$ and $y=(1,-1)$ and look at the inequality of Lemma 1 . We have $\|x+y\|=\|x-y\|=2$ while $\|x\|$ $=\|y\|=2^{1 / q}$. For $p \leqq 2$ we must have $2 \cdot 2^{p} \leqq 2 \cdot 2 \cdot 2^{p / q}$, i.e. $q \leqq p^{\prime}$. For $p \geqq 2$ the inequality is $2 \cdot 2^{p} \leqq 2^{p-1} \cdot 2 \cdot 2^{p / q}$, i.e. $q \leqq p$. Therefore $q \leqq \max \left(p, p^{\prime}\right)$. With $x=(1,0)$ and $y=(0,1)$ we get $q \geqq \min \left(p, p^{\prime}\right)$.

COROLlaRY. $\mathscr{B}_{2}=$ Hilbert spaces.

Proof. For $p=2$, the inequality of Lemma 1 forces equality.

Lemma 2. Suppose $2 \leqq p<\infty$ and $B \in \mathscr{B}_{p}$. Then for $x, h \in B$ with $\|x\|=1$ and $\|h\| \leqq 1$ we have

$$
\|x+h\|^{p}+\|x-h\|^{p}-2\|x\|^{p} \leqq 2^{p-2} p(p-1)\|h\|^{2} .
$$

Proof. Let $\alpha$ with $0<\alpha<1$ be given. Then there exists $\beta$ with $0<\beta<2$ such that the maximum, $\gamma$, of $|\xi+\alpha \eta|^{p}+|\xi-\alpha \eta|^{p}+|\beta \eta|^{p}$ on the "sphere" $\xi^{p}+\eta^{p}=1$ is 
achieved at $\xi=\eta=2^{-1 / p}$. Then $(\xi, \eta) \rightarrow \gamma^{-1}(\xi+\alpha \eta, \xi-\alpha \eta, \beta \eta)$ is a morphism $L_{p}(2, R) \rightarrow L_{p}(3, R)$. Hence if $B \in \mathscr{B}_{p}$ and $x, y \in B$ with $\|x\|=1=\|y\|$ we must have

$$
\|x+\alpha y\|^{p}+\|x-\alpha y\|^{p}+\|\beta y\|^{p} \leqq(1+\alpha)^{p}+(1-\alpha)^{p}+\beta^{p} .
$$

Putting $h=\alpha y$ gives

$$
\|x+h\|^{p}+\|x-h\|^{p}-2 \leqq(1+\|h\|)^{p}+(1-\|h\|)^{p}-2 .
$$

COROLlaRY. $\mathscr{L}_{q} \cap \mathscr{B}_{p}=\{0, R\}$ if $q<2 \leqq p<\infty$.

Proof. It suffices to show that $L_{q}(2, R) \notin \mathscr{B}_{p}$. Take $x=(1,0)$ and $h=(0, \alpha)$. The inequality of the lemma gives

$$
2\left(1+\alpha^{q}\right)^{p / q}-2 \leqq 2^{p-2} p(p-1) \alpha^{2}
$$

which cannot hold for small $\alpha$ since $\left(1+\alpha^{q}\right)^{p / q}-1 \sim(p / q) \alpha^{q}$ as $\alpha \rightarrow 0$.

Lemma 3. For $B \in \mathscr{B}_{p}, 1<p<\infty$, the function $x \mapsto\|x\|^{p}$ is differentiable on $B$.

Proof. Given $x, u \in B, \lim _{t \rightarrow 0+} t^{-1}\left\{\|x+t u\|^{p}-\|x\|^{p}\right\}=F(x ; u)$ always exists. The problem is to show that for each $x \in B, F(x ; \cdot)$ is a real-linear function on $B$. We henceforth regard $B$ as a real Banach space. The proof of Lemma 2 shows that $F(x ;-u)=-F(x ; u)$ for all $x, u \in B$. Consider the matrix

$$
M=\left[\begin{array}{rrr}
1 & -1 & -1 \\
1 & 1 & 0 \\
1 & 0 & 1
\end{array}\right]
$$

as an element of $\operatorname{HOM}\left(L_{p}(3 ; C), L_{p}(3 ; C)\right)$. When $p=1,\|M\|=3$, when $p=2$, $\|M\|=3^{1 / 2}$. Hence by the Riesz Convexity Theorem, $3^{-1 / p} M$ gives a morphism $L_{p}(3 ; R) \rightarrow L_{p}(3 ; R)$ for $1 \leqq p \leqq 2$; that is to say

$$
\|x-y-z\|^{p}+\|x+y\|^{p}+\|x+z\|^{p} \leqq 3\left(\|x\|^{p}+\|y\|^{p}+\|z\|^{p}\right) .
$$

Putting $y=t u, z=t v$ we get

$$
\|x-t(u+v)\|^{p}+\|x+t u\|^{p}+\|x+t v\|^{p}-3\|x\|^{p} \leqq 3 t^{p}\left(\|u\|^{p}+\|v\|^{p}\right) .
$$

Therefore, for $1<p \leqq 2$ we get $F(x ;-u-v)+F(x ; u)+F(x ; v) \leqq 0$; and since $F(x ; \cdot)$ is odd it must be linear. An argument similar to that of Lemma 2 shows that $|F(x ; u)| \leqq p\|x\|^{p-1}\|u\|$. Since $F(x ; x)=p\|x\|^{p}$ and $B$ is uniformly convex, it follows that $x \rightarrow p^{-1} F(x ; \cdot)$ is a one-to-one map of the unit sphere of $B$ into the unit sphere of $B^{\prime}$. Since $B^{\prime}$ is uniformly convex, this map must be onto (the argument is this: given $\xi \in B^{\prime}$ with $\|\xi\|=1$ there exists $x \in B$ with $\|x\|=1$ and $\langle x, \xi\rangle=1$; put $\eta=p^{-1} F(x ; \cdot)$; then $\left\langle x, \frac{1}{2}(\xi+\eta)\right\rangle=1$ so $\left\|\frac{1}{2}(\xi+\eta)\right\|=1$ which forces $\left.\xi=\eta\right)$. For $\xi, \eta \in B^{\prime}$ put

$$
\Phi(\xi ; \eta)=\lim _{t \rightarrow 0+} t^{-1}\left\{\|\xi+t \eta\|^{p^{\prime}}-\|\xi\|^{p^{\prime}}\right\}
$$


If $\xi$ is on the unit sphere in $B^{\prime}$ then $\xi=p^{-1} F(x ; \cdot)$ for some $x \in B$. One can establish that $\Phi(\xi ; \eta)=p^{\prime}\langle x, \eta\rangle$, and hence $\xi \rightarrow\|\xi\|^{p^{\prime}}$ gives a differentiable function on $p^{\prime}$. This allows me to conclude the assertion of the lemma for $2<p<\infty$.

4. Sheaves and elementarity. Let $X$ be a paracompact Hausdorff space and $\mathscr{B}$ a complete category. Put $\tau^{*}(X)$ for the category of reverse inclusions of open subsets of $X$. Let $F: \tau^{*}(X) \rightarrow \mathscr{B}$ be a covariant functor. For each $x \in X$ put $F_{x}$ $=$ ind $\lim _{\mathscr{N}_{x}} F$ where $\mathscr{N}_{x}$ is a fundamental system of neighborhoods of $x$. The functor $F$ is a $\mathscr{B}$-valued sheaf over $X$ if

(S1) Let $\mathscr{A}$ be any collection of open subsets of $X$ and $\mathscr{A}^{*}$ the subcategory of $\tau^{*}(X)$ constituted by the morphisms $U \supset U \cap V$ for $U, V \in \mathscr{A}$; then if $A$ is the union of the elements of $\mathscr{A}, F(A)$ with the morphisms $F(A) \rightarrow F(U), U \in \mathscr{A}$ gives a projective limit, proj $\lim _{\mathscr{A}^{*}} F$.

(S2) For each open set $U$ in $X$ the canonical morphism $F(U) \rightarrow \prod_{x \in U} F_{x}$ an extremal monomorphism.

If $X \stackrel{\varphi}{\rightarrow} Y$ is a continuous map of paracompact Hausdorff spaces and $F$ is a sheaf over $X$ we obtain a sheaf over $Y$ by defining $\varphi F: \tau^{*}(Y) \rightarrow \mathscr{B}$ as $\varphi F(V)$ $=F\left(\varphi^{-1}(V)\right)$. The sheaf $\varphi F$ is called the direct image of $F$.

A class of objects of $\mathscr{B}$ will be called semi-elementary if it is stable for isomorphism and has the property: if $F$ is a $\mathscr{B}$-valued sheaf over a discrete space $X$ each of whose stalks $F_{x}$ belongs to the class and $\beta: X \rightarrow \check{X}$ is the map of $X$ into its Čech compactification then all the stalks of the direct image $\beta F$ belong to the class. A stalk of the form $(\beta F)_{y}$ where $y \in \check{X}$ is called an ultraproduct. Presumably, semielementary classes are defined by properties of a special logical form appropriate to the category in question.

In the case where $\mathscr{B}$ is the category of Banach spaces and $F$ is a $\mathscr{B}$-valued sheaf over $X$, for each element $f \in F(U)$ we put $f(x)$ for the value of $f$ under the canonical morphism $F(U) \rightarrow F_{x}$ where $x \in U$. According to (S2), $\|f\|_{U}=\sup _{x \in U}|f(x)|$ where $\|f\|_{U}$ is the norm in $F(U)$ and $|f(x)|$ is the norm in $F_{x}$. If $U \supset V$ we shall write $\|f\|_{V}$ for the norm of the image of $f$ under $F(U) \rightarrow F(V)$. Given $x \in X$ and $b \in F_{x}$ there exists a decreasing sequence $\left\{U_{n}\right\}$ of neighborhoods of $x$ and elements $f_{n} \in F\left(U_{n}\right)$ such that $\lim _{N \rightarrow \infty} f_{n}(x)=b$ and for each $\varepsilon>0$ there exists an integer $m$ such that $\left\|f_{m}-f_{n}\right\|_{U_{n}}<\varepsilon$ whenever $n>m$. It follows that $x \mapsto|f(x)|$ is an upper semicontinuous function on $U$ when $f \in F(U)$; indeed $|f(x)|=\inf _{V \in \mathscr{N}_{x}} \sup _{y \in V}|f(y)|$.

In case $X$ is a discrete space, $\check{X}$ the Čech compactification, and $F$ a Banach-valued sheaf over $X$ we must have for each open $V \subset \check{X}$ and $g \in \beta F(V)$ that $y \mapsto|g(y)|$ is a continuous function on $V$. It is an immediate consequence of Proposition 0 that $\mathscr{B}_{p}$ is a semi-elementary class of Banach spaces. What is much deeper is that $\mathscr{L}_{p}$ is a semi-elementary class in the category of real Banach spaces. This follows from the work of Nakano [8] where the basic category is Banach lattices, but Banachlattice ultraproducts coincide with the Banach-space ultraproducts. (Note: $\{\boldsymbol{R}\}$ is semi-elementary in real Banach spaces but not in real vector spaces; the norm- 
function on a Banach space kills infinitesimals.) For complex $\mathscr{L}_{p}$-spaces it is not known whether the class is semi-elementary, much less is there a concrete "elementary" characterization.

Bretagnolle, Dacunha-Castelle, and Krivine [3] proved that the class of subspaces of real $\mathscr{L}_{p}$-spaces is stable for direct unions by observing that $\mathscr{L}_{p}$ was semielementary and then using a concrete form of the following argument. A category $\mathscr{B}$ is said to have the "Grothendieck property" if whenever $\mathscr{D}$ is a directed set and $F, G: \mathscr{D} \rightarrow \mathscr{B}$ are functors connected by natural extremal monomorphisms $F \rightarrow G$ then the natural morphisms ind $\lim _{\mathscr{D}} F \rightarrow$ ind $\lim _{\mathscr{D}} G$ is an extremal monomorphism. Banach spaces have the Grothendieck property.

Proposition 8. Let $\mathscr{E}$ be a semi-elementary class in a complete category with the Grothendieck property. Let $\mathscr{H} \mathscr{E}$ be the class of subobjects of objects of $\mathscr{E}$. The $\mathscr{H} \mathscr{E}$ is semi-elementary and stable for direct unions.

Proof. That $\mathscr{H} \mathscr{E}$ is semi-elementary is an obvious consequence of the Grothendieck property and the fact that projective limits, in particular products, always preserve extremal monomorphisms. Now let $\mathscr{D}$ be a directed set and $\Phi: \mathscr{D} \rightarrow \mathscr{B}$ a functor such that for each $x \in \mathscr{D}, \Phi(x) \in \mathscr{H} \mathscr{E}$. We suppose that when $x \prec y$, $\Phi(x) \rightarrow \Phi(y)$ is an extremal monomorphism; then ind $\lim _{\mathscr{D}} \Phi$ is a direct union. Regard $\mathscr{D}$ as a discrete space and define $F: \tau^{*}(\mathscr{D}) \rightarrow \mathscr{B}$ by $F(u)=\prod_{x \in U} \Phi(x)$. When $U \supset V$ the morphism $F(U) \rightarrow F(V)$ is projection on a partial product. For the stalks of the sheaf $F$ there is a natural identification of $F_{x}$ with $\Phi(x)$. Let $\mathscr{N}$ be an ultrafilter of cofinal subsets of $\mathscr{D}$. Then there is a point $c \in \check{\mathscr{D}}$, the Čech compactification, such that $\mathscr{N}$ is the trace on $\mathscr{D}$ of the open neighborhoods of $c$. Put $\Gamma$ $=(\beta F)_{c}=$ ind $\lim _{\mathscr{N}} F$. We have $\Gamma \in \mathscr{H} \mathscr{E}$ since $\mathscr{H} \mathscr{E}$ is semi-elementary. Write $(\mathscr{N}, \mathscr{B})$ for the category of functors from the directed set $\mathscr{N}$ to $\mathscr{B}$. Given $x \in \mathscr{D}$, put $S_{x}=\{y \in \mathscr{D}: x \prec y\}$ and define a functor $G: \mathscr{D} \rightarrow(\mathscr{N}, \mathscr{B})$ by $G(x)(U)$ $=F\left(U \cap S_{x}\right)$, the morphism $G(x) \rightarrow G(y)$ when $x \prec y$ being the natural partial product projections. We may regard ind $\lim _{\mathscr{N}}$ as a functor from $(\mathscr{N}, \mathscr{B})$ to $\mathscr{B}$ and hence ind $\lim _{\mathscr{N}} G$ as a functor from $\mathscr{D}$ to $\mathscr{B}$. Because of the way $\mathscr{N}$ was chosen, $\left\{U \cap S_{x}: U \in \mathscr{N}\right\}$ is cofinal in $\mathscr{N}$ for each $x \in \mathscr{D}$. Thus there is a natural identification of ind $\lim _{\mathscr{N}} G$ with $\Gamma$. Write $\Phi^{*}: \mathscr{D} \rightarrow(\mathscr{N}, \mathscr{B})$ for the functor $\Phi^{*}(x)(U)$ $=\Phi(x)$. There is a natural transformation $\Phi^{*} \rightarrow G$ coming from $\Phi(x) \rightarrow \prod_{x \prec y \in U} \Phi(y)$ as a product of morphisms $\Phi(x) \rightarrow \Phi(y)$. For fixed $x \in \mathscr{D}, \Phi^{*}(x) \rightarrow G(x)$ are natural extremal monomorphisms. By the Grothendieck property, ind $\lim _{\mathscr{N}} \Phi^{*}(x) \rightarrow$ ind $\lim _{\mathscr{N}} G(x)$ is an extremal monomorphism, but this is simply a natural transformation $\Phi \rightarrow \Gamma$. Once again by the Grothendieck property, ind $\lim _{\mathscr{D}} \Phi \rightarrow$ ind $\lim _{\mathscr{D}} \Gamma=\Gamma$ is an extremal monomorphism, i.e. the direct union ind $\lim _{\mathscr{D}} \Phi$ is a subobject of $\Gamma \in \mathscr{H} \mathscr{E}$, hence the direct union is in $\mathscr{H} \mathscr{E}$.

\section{BIBLIOGRAPHY}

1. S. Bochner, Integration von Funktionen, deren Werte die Elemente eines Vectorraumes sind, Fund. Math. 20 (1933), 262-276. 
2. S. Bochner, Stable laws of probability and completely monotone functions, Duke Math. J. 3 (1937), 726-728.

3. J. Bretagnolle, D. Dacunha-Castelle, and J.-L. Krivine, Lois stables et espaces $L^{p}$, Ann. Inst. H. Poincaré Sect. B 2 (1965/66), 231-259. MR 34 \#3605.

4. J. A. Clarkson, Uniformly convex spaces, Trans. Amer. Math. Soc. 40 (1936), 396-414.

5. C. S. Herz, A class of negative-definite functions, Proc. Amer. Math. Soc. 14 (1963), 670676. MR 28 \#1477.

6. P. Lévy, Théorie de l'addition des variables aléatoires, Gauthier-Villars, Paris, 1937.

7. J. Marcinkiewicz and A. Zygmund, Quelques inégalités pour les opèrations linèaires, Fund. Math. 32 (1939), 115-121.

8. H. Nakano, Uber normierte teilweisegeordnete Moduln, Proc. Imp. Acad. Tokyo 17 (1941), 311-317. MR 7, 249.

BRANDEIS UNIVERSITY,

Waltham, MassachusetTs 02154 